\title{
Gastro Jejunal Inner Lumen Bypass Device Inhibits the Growth of Pigs
}

Hidetoshi Matsunami ${ }^{1-3}$, Noriko Sasaki ${ }^{2}$, Tomomi Yoshikawa ${ }^{*}$, Masao Takemura ${ }^{2,3}$, Kazuhiro Watanabe ${ }^{4}, 5$, Sanae Shibata ${ }^{4}$, Mifumi Kawabe ${ }^{4}$, Tatsuya Matsubara $^{5}$, Yuki Murakami ${ }^{6}$ and Makoto Hayashi ${ }^{2}$

${ }^{1}$ Department of Surgery, Sosaikouseikai Clinical Foundation Matsunami General Hospital, Gifu 501-6062, Japan

${ }^{2}$ Matsunami Research Park, Sosaikouseikai Clinical Foundation Matsunami General Hospital, Gifu 501-6062, Japan

${ }^{3}$ Human Health Sciences, Graduate School of Medicine and Faculty of Medicine, Kyoto University, Kyoto 606-8507, Japan

${ }^{4}$ Joint Department of Veterinary Medicine, Faculty of Applied Biological Sciences, Gifu University, Gifu 501-1193, Japan

5 United Graduate school of Veterinary Sciences, Gifu University, Gifu 501-11193, Japan

${ }^{6}$ Organization for Research Initiatives and Development, Doshisha University, Kyoto 602-8580, Japan

*Corresponding author: Tomomi Yoshikawa, Research Park, Sosaikouseikai Clinical Foundation Matsunami General Hospital, 185-1 Dendai Kasamatsu-cho, Hashimagun, Gifu 501-6062, Japan, Tel: +81-58-388-0111; E-mail: ytomomi@matsunami-hsp.or.jp

Rec Date: Nov 05, 2015; Acc Date: Jan 18, 2016; Pub Date: Jan 25, 2016

Copyright: (c) 2016, Matsunami H, et al. This is an open-access article distributed under the terms of the Creative Commons Attribution License, which permits unrestricted use, distribution, and reproduction in any medium, provided the original author and source are credited.

\begin{abstract}
The EndoBarrier has a weak point caused by a Teflon membrane, which completely inhibits the movement of water and food. We developed a new type of gastro jejunal inner lumen bypass device (GJB) using an artificial net that has a hole instead of a membrane.

We inserted a net in the gastric antrum-duodenum-jejunum via open laparotomy. The growth of pigs with long nets was suppressed compared with the sham operation. However, there were no differences in blood biochemistry or anatomical findings at autopsy between the groups. As a result, GJB can control the growth of pigs without requiring dietary restriction.
\end{abstract}

Keywords: Bariatric surgery; EndoBarrier; Gastro jejunal inner lumen bypass devices (GJB)

\section{Introduction}

Obesity is a risk factor for cardiovascular disease, type II diabetes, stroke, cancer and Alzheimer's disease [1-5]. Obese individuals need to lose weight. However, weight loss attempts are frequently unsuccessful. As a result, surgical treatment has emerged as an effective treatment for patients with obesity. Commonly performed procedures include Rouxen-Y gastric bypass, sleeve gastrectomy, and biliopancreatic diversion/ duodenal switch [6]. However, several short- and long-term complications may occur with these procedures, including anastomotic leaks, bleeding, infection, dumping syndrome and malabsorption of micronutrients (e.g., iron, calcium, and vitamins). Furthermore, the procedure is irreversible [7,8]. As a result, at the time of biliary and pancreatic carcinoma surgery, future reconstruction may be very complicated.

To avoid an irreversible procedure, several recent human studies have used the EndoBarrier Gastrointestinal Liner, which is a $60 \mathrm{~cm}$ impermeable fluoropolymer, Teflon membrane that is fixated to the duodenum and extends into the small intestine; it usually terminates in the proximal jejunum. The EndoBarrier is minimally invasive and fully reversible and is effective for weight loss; it can also improve diabetes (greater reductions in glycated hemoglobin; HbAlc) [9-12]. However, on 31 July 2015, a medical device company and the U.S. Food and Drug Administration (the FDA) announced the decision to discontinue the U.S. EndoBarrier therapy trial for the treatment of obese patients because of a higher than anticipated incidence of hepatic abscesses from bacterial infections of the liver (GI Dynamics Press Release - July 30, 2015, GI Dynamics Concludes ENDO Trial).
To solve these problems, we developed new type of gastro jejunal inner lumen bypass device (GJB) with a longer length using an artificial net. We have a patent pending (2014-068503, PTC/ JP2015/57361).

The difference between the Teflon membrane of the EndoBarrier and our GJB net is that our net has holes, allowing for free movement of water and food at the surface of the intestinal mucosa, indicating that digestion and absorption are not completely inhibited at that location. So it may prevent obstruction of the ampulla of Vater which impairs the flow of bile and causes hepatic abscess from the bacterial infection of the liver.

\section{Methods}

Four-to-five-week-old male Landrace, Large white and Duroc (LWD) pigs with an average body weight of 7-8 $\mathrm{kg}$ from Seiko animal industry Co., Ltd. (Gifu, Japan) were included in this study. Pigs were housed in separate pens at a constant temperature under a light-dark cycle. They had free access to food, semi-solid animal feed (for growing animals) (Winny Cline, NOSAN Co., Yokohama, Japan), and water. The pigs were fed the same amount of feed, which was based on the Japanese Feeding Standard for pigs (Japan Livestock Industry Association, 2005), every day. All pig experiments were approved by the university committee on animal research, and the pigs received humane care in accordance with the National Institutes of Health publication 86-23, the "Guide for the Care and Use of Laboratory Animals."

Laparotomy, gastrostomy and enterotomy were performed, and a catheter (New Enteral feeding tube, COVIDIENTM, Co., Tokyo, Japan) was inserted into the jejunum and extended into the gastric 
Citation: Matsunami H, Sasaki N, Yoshikawa T, Takemura M, Watanabe K, et al. (2016) Gastro Jejunal Inner Lumen Bypass Device Inhibits the Growth of Pigs. J Diabetic Complications Med 1: 103.

antrum of the stomach. The end of the GJB was then connected to the introduction catheter, and the catheter was removed from the enterotomy (Figure 1). The oral side of the GJB was fixed to the fullthickness gastric wall by non-absorbable prolene 2-0 suture (4-8 stitches). The jejunum side of the GJB was also weakly fixed by absorbable vicryl 5-0 suture (1-2 stitches). Finally, the laparotomy, gastrostomy and enterotomy were completed, and the area was irrigated with saline. The sham operation consisted of laparotomy and gastrotomy with repair. Control pig was bred without undergoing an operation during the same period. After surgery, the pigs were provided free access to water and food after approximately $15 \mathrm{~h}$.

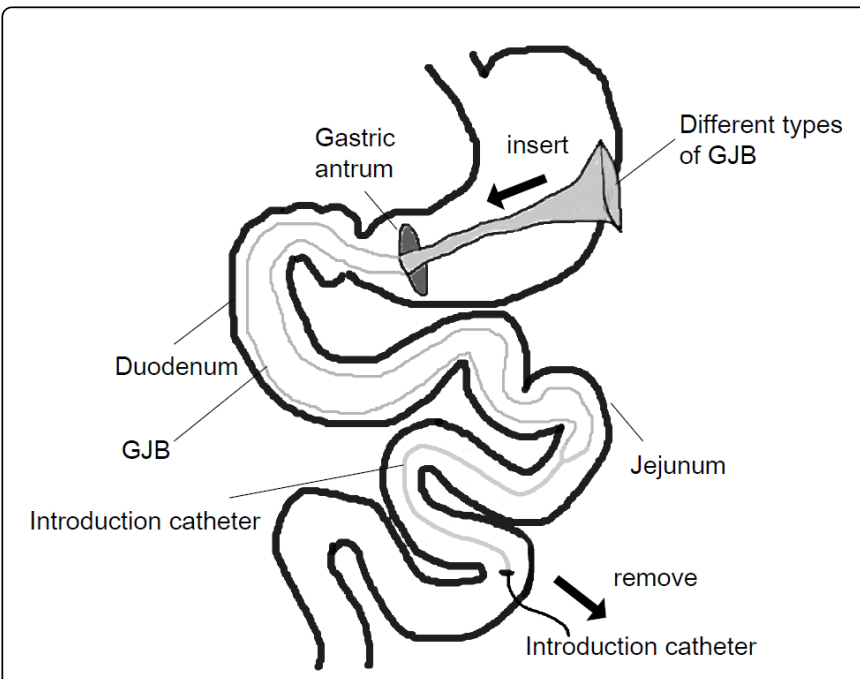

Figure 1: How to insert the GJB.

The following nets were used in this study for the GJB: a plastic net, which is used as an orange package in Japan (made of polyethylene, Morishita Inc., Okayama, Japan, 5-10 mm hole); knit fabric net, which is used as nylon stockings (ATSUGI Co., Ltd., Kanagawa, Japan, 1-2 $\mathrm{mm}$ hole); and a nonwoven net, which farmers initially used to keep insects off plants (made of polypropylene, Dio Chemicals, Ltd., Tokyo, Japan, less than $1 \mathrm{~mm}$ hole) (Figure 2). The diameter of the net was 20 $\mathrm{mm}$, and the lengths of the short nets were as follows: plastic net (45 $\mathrm{cm})$, knit fabric net $(70 \mathrm{~cm})$, and nonwoven net $(45 \mathrm{~cm})$. The lengths of the long net were as follows: plastic net $(115 \mathrm{~cm})$, knit fabric net (109 $\mathrm{cm})$, and nonwoven net $(125 \mathrm{~cm})$. Every week after surgery, we checked the animals' body weights (Figure 3). Additionally, serum total protein, albumin (Alb), total cholesterol, triglycerides (TG), highdensity lipoprotein (HDL), low-density lipoprotein (LDL), magnesium $(\mathrm{Mg})$ and calcium (Ca) were measured by standard laboratory methods (Table 1). After five weeks, the pigs were killed and autopsied, and we observed the intraperitoneal cavity, the inside of the stomach and the intestine.
(1)

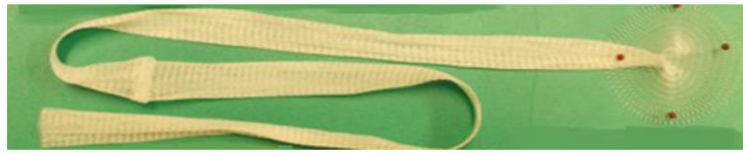

(2)

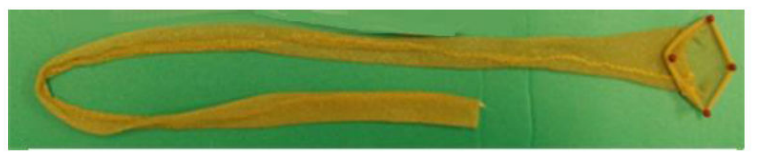

(3)

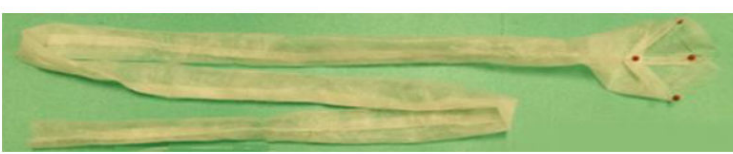

Figure 2: The different GJB types. (1) Plastic net (Short $45 \mathrm{~cm}$, Long $115 \mathrm{~cm}$ ). (2) Knit fabric net (Short $70 \mathrm{~cm}$, Long $109 \mathrm{~cm}$ ). (3) Nonwoven net (Short $45 \mathrm{~cm}$, Long $125 \mathrm{~cm}$ ).

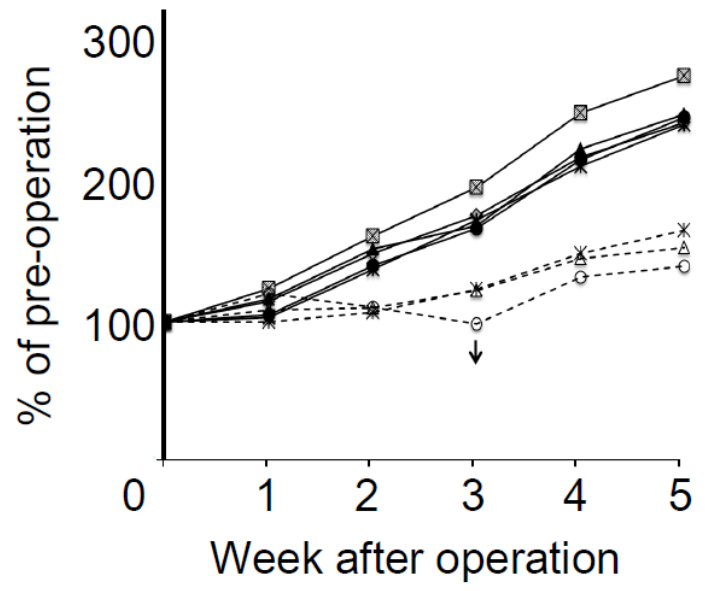

\footnotetext{
$-8-$ Control $\quad-$ Sham-operated

$\neg$ Plastic net (Short: $45 \mathrm{~cm}$ ) - $A$ - Plastic net (Long: $115 \mathrm{~cm}$ )

- - Knit fabric net (Short: $70 \mathrm{~cm}$ ) $-\odot-$ Knit fabric net (Long: $109 \mathrm{~cm}$ )

$\rightarrow \leftarrow$ Nonwoven net (Short: $45 \mathrm{~cm}$ ) - * - Nonwoven net (Long: $125 \mathrm{~cm}$ )

$\downarrow$ Shed in stool
}

Figure 3: The body weight change observed in the pigs.

\section{Results}

After five weeks, the control pig body weight was nearly three times the weight on day 0 . Pig undergoing the sham operation had a growth rate depicted in (Figure 3). 
Citation: Matsunami H, Sasaki N, Yoshikawa T, Takemura M, Watanabe K, et al. (2016) Gastro Jejunal Inner Lumen Bypass Device Inhibits the Growth of Pigs. J Diabetic Complications Med 1: 103.

Page 3 of 4

\section{The short net inserted pigs}

When we conducted the autopsy, the short plastic net $(45 \mathrm{~cm})$ and short nonwoven net $(45 \mathrm{~cm})$ flowed back from the jejunum into the stomach. The short knit fabric net $(70 \mathrm{~cm})$ could not be found inside the stomach or intestine; therefore, we concluded that it may have been shed in the stool.

In the three pigs receiving short net insertions, there were no apparent abnormal findings, such as ulcers of the stomach or intestine, adhesion, or ileus. The body weight of the pigs with the short net increased approximately 2.5 -fold at the end of five weeks. It appears there were no differences in the body weight increases of the pigs inserted with short nets compared with the sham-operated pig (Figure 3).

\section{The long net inserted pigs}

The long knit fabric net $(109 \mathrm{~cm})$ was excreted into the stool three weeks after the operation (technical error of stitches in the stomach). The long plastic and nonwoven nets slightly prevented body weight gain with an approximately $60 \%$ growth suppression compared with the sham-operated pig after five weeks.

On autopsy, administered food was observed on the outside and inside of the net. No abnormal findings were seen inside or outside of the intestine. There were no differences in body weight changes, regardless of the hole size, nor were there any differences in blood chemistry results (Table 1).

\begin{tabular}{|c|c|c|c|c|c|c|c|c|c|}
\hline & & Total & & & & & & & \\
\hline & & protein & & & & NLL & LDL & ing & da \\
\hline & & g/dl & g/dl & $\mathrm{mg} / \mathrm{dl}$ & $\mathrm{mg} / \mathrm{dl}$ & $\mathrm{mg} / \mathrm{dl}$ & $\mathrm{mg} / \mathrm{dl}$ & $\mathrm{mg} / \mathrm{dl}$ & $\mathrm{mg} / \mathrm{dl}$ \\
\hline & Pre-operation & 4.8 & 3.3 & 109 & 51 & 49.6 & 48 & 2.4 & 11.7 \\
\hline & 5 weeks & 4.7 & 3.6 & 75 & 45 & 34.9 & 30 & 1.8 & 11.1 \\
\hline & Pre-operation & 5 & 3.6 & 124 & 66 & 56.4 & 55 & 2.9 & 12.9 \\
\hline & 5 weeks & 4.8 & 4.1 & 69 & 19 & 29.8 & 30 & 1.9 & 11.2 \\
\hline Eahrir net (c) & Pre-operation & 4.3 & 3.2 & 79 & 23 & 42.5 & 28 & 2.2 & 12.1 \\
\hline & 5 weeks & 4.9 & 3.9 & 73 & 26 & 32.6 & 30 & 2 & 12 \\
\hline & Pre-operation & 4.4 & 3.1 & 91 & 27 & 49.9 & 36 & 2.5 & 11.9 \\
\hline & 5 weeks & 4.9 & 3.4 & 68 & 16 & 29.7 & 29 & 1.7 & 11.1 \\
\hline & Pre-operation & 4.8 & 3 & 93 & 12 & 36.6 & 48 & 2.43 & 11.8 \\
\hline & 5 weeks & 5.6 & 3.5 & 88 & 18 & 38.6 & 43 & 2.35 & 12.43 \\
\hline & Pre-operation & 5.2 & 3.7 & 133 & 74 & 45 & 78 & 2.9 & 12.2 \\
\hline & 5 weeks & 5.1 & 3.4 & 95 & 73 & 42.3 & 43 & 2.5 & 11.6 \\
\hline Ctocking & Pre-operation & 4.9 & 3.3 & 147 & 85 & 56.3 & 76 & 2.4 & 11.7 \\
\hline & 5 weeks & 5.5 & 4.1 & 114 & 118 & 56.2 & 44 & 2.4 & 11.3 \\
\hline & Pre-operation & 4.5 & 3 & 150 & 27 & 42.8 & 96 & 2.7 & 10.7 \\
\hline & 5 weeks & 5.2 & 3.3 & 116 & 43 & 44.9 & 60 & 1.9 & 11.3 \\
\hline
\end{tabular}

Table 1: Blood biochemistry.

\section{Discussion}

The purpose of the bariatric procedure and EndoBarrier treatment is to reduce body weight in obese people. However, in this study, due to the space constraints of our laboratory, we investigated the effect of growth inhibition on younger pigs instead of conducting research on obese pigs.

The length of the EndoBarrier Gastrointestinal Liner is only $60 \mathrm{~cm}$. It covers only $35 \mathrm{~cm}$ of the small intestine. The length of the device may not be sufficient. Furthermore, the EndoBarrier Gastrointestinal Liner is made of the Teflon, which may be a point of weakness. Teflon completely inhibits the movement of water and food, and it may obstruct the ampulla of Vater, preventing the flow of bile and causing a liver abscess. To solve these problems, we envisioned a porous membrane. However, we used nets with different hole sizes (ranging from less than $1 \mathrm{~mm}$ to $10 \mathrm{~mm}$ ) as the gastro jejunal inner lumen bypass device.

We found that long GJB-inserted pigs demonstrated a growth suppression of approximately $60 \%$ compared with sham-operated pig. However, compared with the plastic net $(5-10 \mathrm{~mm})$ and nonwoven net (less than $1 \mathrm{~mm}$ ), there was no difference in growth suppression, regardless of the hole diameter. One reason may be that the food the pigs ate is similar to rice gruel. It is easily softened with water and 
Citation: Matsunami H, Sasaki N, Yoshikawa T, Takemura M, Watanabe K, et al. (2016) Gastro Jejunal Inner Lumen Bypass Device Inhibits the Growth of Pigs. J Diabetic Complications Med 1: 103.

Page 4 of 4

gastric juice. Therefore, regardless of the sizes of the holes, the food can freely move inside and outside of the net.

On the other hand, there were no differences in blood biochemistry (total protein, albumin, total cholesterol, etc.) between the shamoperated and operated pigs. Therefore, the GJB net seems to inhibit digestion and absorption in a physiologic manner.

This study is the first to demonstrate that inserting different types of artificial nets into the gastro jejunal area inhibits the growth of pigs. These results demonstrate that GJB nets can promote weight loss without requiring dietary restriction.

\section{References}

1. Ng M, Fleming T, Robinson M, Thomson B, Graetz N, et al. (2014) Global, regional, and national prevalence of overweight and obesity in children and adults during 1980-2013: a systematic analysis for the Global Burden of Disease Study 2013. Lancet 384: 766-781

2. Hanson C, Rutten EP, Wouters EF, Rennard S (2014) Influence of diet and obesity on COPD development and outcomes. Int J Chron Obstruct Pulmon Dis 9: 723-733.

3. Finkelstein EA, Fiebelkorn IC, Wang G (2003) National medical spending attributable to overweight and obesity: how much, and who's paying? Health Aff (Millwood) Suppl Web Exclusives: W3-219-26.

4. Kaplan LM (2005) Gastrointestinal management of the bariatric surgery patient. Gastroenterol Clin North Am 34: 105-125.
5. Buchwald H, Avidor Y, Braunwald E, Jensen MD, Pories W, et al. (2004) Bariatric surgery: a systematic review and meta-analysis. JAMA 292: 1724-1737.

6. Buchwald H, Oien DM (2013) Metabolic/bariatric surgery worldwide 2011. Obes Surg. 23: 427-436.

7. Buchwald H, Estok R, Fahrbach K, Banel D, Sledge I (2007) Trends in mortality in bariatric surgery: a systematic review and meta-analysis. Surgery 142: 621-635.

8. Bal BS, Finelli FC, Shope TR, Koch TR (2012) Nutritional deficiencies after bariatric surgery. Nat Rev Endocrinol 8: 544-556.

9. Rohde U, Hedback N, Gluud LL, Vilsboll T, Knop FK (2013) Effect of the EndoBarrier Gastrointestinal Liner on obesity and type 2 diabetes: protocol for systematic review and meta-analysis of clinical studies. BMJ Open 3: e003417.

10. Patel SR, Mason J, Hakim N (2012) The Duodenal-Jejunal Bypass Sleeve (EndoBarrier Gastrointestinal Liner) for Weight Loss and Treatment of Type II Diabetes. Indian J Surg 74: 275-277.

11. Koehestanie P, de Jonge C, Berends FJ, Janssen IM, Bouvy ND, et al. (2014) The Effect of the Endoscopic Duodenal-Jejunal Bypass Liner on Obesity and Type 2 Diabetes Mellitus, a Multicenter Randomized Controlled Trial. Ann Surg 260: 984-992.

12. Schouten R, Rijs CS, Bouvy ND, Hameeteman W, Koek GH, et al. (2010) A multicenter, randomized efficacy study of the EndoBarrier Gastrointestinal Liner for presurgical weight loss prior to bariatric surgery. Ann Surg 251: 236-243. 